\title{
Neuroimaging Advances in Neurologic and Neurodegenerative Diseases
}

\author{
Shannon L. Risacher ${ }^{1} \cdot$ Andrew J. Saykin ${ }^{1}$ \\ Accepted: 6 August 2021 / Published online: 19 August 2021 \\ (c) The American Society for Experimental NeuroTherapeutics, Inc. 2021
}

Neuroimaging tools, including magnetic resonance imaging (MRI), positron emission tomography (PET), and others (computerized tomography (CT), single-photon emission computerized tomography (SPECT), etc.) can be important biomarkers for identification, tracking, diagnosis, and treatment monitoring of neurologic diseases. Widely used in neurodegenerative disorders like Alzheimer's disease (AD), frontotemporal lobar degeneration (FTLD), and Parkinson's disease (PD) and associated disorders, neuroimaging methods are also applied to many other neurologic diseases to uncover important information about underlying biology, diagnostic classification, and treatment response. The most commonly used neuroimaging techniques for neurodegenerative disorders are MRI and PET, which are further described in this issue of Neurotherapeutics by C. Schwarz (2020) [1]. Although Dr. Schwarz's review is largely focused on neurodegenerative diseases, the methodologies described are generally applicable to all types of neurologic disease.

In neurodegenerative diseases, we observe significant patterns of atrophy on MRI that are generally disease-type specific. In addition, the advent of PET tracers that target two of the major proteinopathies in $\mathrm{AD}$ and other diseases, amyloid and tau, has allowed for early detection, monitoring of disease progression, and disease-specific target outcomes in clinical trials. Findings and future directions of studies of neuroimaging biomarkers in $\mathrm{AD}$ are explored in the review by Buckley (2020) [2]. Unfortunately, many other proteinopathies, such as TDP-43, a major cause of FTLD, and alphasynuclein, a major cause of PD, PD dementia, and Lewy body dementia (LBD), are not yet able to be visualized using PET. In these cases, MRI is the primary tool used to assess changes in TDP-43-related dementia and alpha-synucleinassociated dementias. Findings from MRI and other imaging

Shannon L. Risacher

srisache@iupui.edu

1 Department of Radiology and Imaging Sciences, Indiana University School of Medicine, Indianapolis, IN, USA tools from the different FTLD forms and the link of these differences to the underlying pathology (i.e., tau, TDP-43, etc.) are explored by Peet et al. (2021) [3]. This review captures the variability and heterogeneity in FTLD and their links to imaging patterns. Finally, Brooks (2020) provides a comprehensive review of neuroimaging in PD and related disorders (such as LBD), focusing on MRI, SPECT (i.e., DATScan), and PET findings (primarily dopamine-focused and other neurotransmitter-focused tracers) [4].

A major goal of neuroimaging tools in studies of neurodegenerative disorders is to define the best markers for therapeutic trials. For example, neuroimaging can be used to screen individuals for those at highest risk to have the pathological target of a therapeutic agent. In addition, neuroimaging tools may be used as secondary, and perhaps one day, primary endpoints of therapeutic trials. A. Schwarz (2020) reviews the use of neuroimaging in therapeutic trials, exploring these issues in depth [5]. Although the focus is on therapeutic trials of neurodegenerative conditions, neuroimaging can be used in this fashion in therapeutic trials for many types of neurologic diseases.

Given the recent approval of aducanamab (Aduheld), a drug which directly targets and clears amyloid from the brain, as a disease-modifying treatment for $\mathrm{AD}$, neuroimaging of amyloid now has clinical relevance to treatment course. Although these are early days, amyloid imaging techniques are at the forefront of the personalized care of $\mathrm{AD}$ and important for determining those eligible for this new drug. However, the prospect of providing amyloid status to individuals with symptoms and even perhaps without symptoms on a large scale brings up many potential ethical questions about returning these results to patients. Wilkenfeld et al. (2020) cover the findings to date on return of results, primarily of amyloid PET results, in both symptomatic and asymptomatic individuals [6]. They suggest that it is the clinician's duty to inform or at least try to provide information to hesitant symptomatic individuals and their loved ones using a transactional model of communication. 
In addition to the sporadic and familial neurodegenerative conditions primarily associated with aging, chronic traumatic encephalopathy (CTE) is a neurodegenerative condition thought to be caused by repetitive head trauma. Studies in individuals with CTE have suggested neurodegenerative changes visible on MRI, with little evidence of binding of tau tracers developed for AD. Neuroimaging and other biomarker studies of CTE are explored by Alosco et al. (2020) [7]. Unfortunately, specific biomarkers for CTE, such as CTE-tau focused PET tracers, are not yet available. Future neuroimaging research in this area will allow for a better understanding of the specific patterns and pathologies associated with repetitive head trauma and hopefully novel treatments to combat CTE.

Other neurologic disorders have been investigated with neuroimaging and other biomarker tools. In particular, neuroimaging studies are commonly used in pre-surgical planning and other studies related to temporal lobe epilepsy, as reviewed by Goodman and Szaflarski (2021) [8]. In addition, research studies investigating changes in brain functional and structural connectivity in patients with epilepsy have allowed for a better understanding of the underlying biological changes associated with disease. Other neuroimaging studies have focused on psychiatric disorders, including anxiety, depression, addiction, and psychosis. Recently, transcranial magnetic stimulation has been proposed as a treatment for and biological probe of underlying pathology in schizophrenia. Findings from neuroimaging studies related to these novel methods of treatment are explored by Brandt and colleagues (2020) [9]. Finally, non-central nervous system (CNS) diseases and associated treatments can show effects in the brain and on cognition. For example, non-CNS cancers and their chemotherapeutic and/or hormonal treatments have been associated with cognitive effects, known as cancer- and treatment-related cognitive dysfunction (CRCD). CRCD has been shown to relate to structural findings on MRI, which are summarized by McDonald (2021) [10]. As development of novel cancer treatments continues, the impact of therapies for cancer and CRCD on neuroimaging-assessed brain structure and function, as well as cognition, is important to consider.

In this issue of Neurotherapeutics, we invited experts in neuroimaging across neurologic diseases to summarize the state of the field and propose steps needed to move the field forward. As medical science has begun to move towards the idea of personalized medicine, where assessments, diagnostics, and therapeutics are tailored to an individual's genetic background, amount and type of pathologic presentation, lifestyle, and other factors, the integration of neuroimaging into decisions about the development and use of targeted therapeutics will continue to advance medical treatment and provide increased efficacy of life-saving treatments.

Supplementary Information The online version contains supplementary material available at https://doi.org/10.1007/s13311-021-01105-7.

Acknowledgements Required Author Forms Disclosure forms provided by the authors are available with the online version of this article.

Funding This work was supported by funding from the National Institute on Aging (NIA; R01 AG061788, K01 AG049050, R01 AG19771, and P30 AG010133).

\section{References}

1. Schwarz CG. Uses of Human MR and PET Imaging in Research of Neurodegenerative Brain Diseases. Neurotherapeutics. 2021. https://doi.org/10.1007/s13311-021-01030-9.

2. Buckley RF. Recent Advances in Imaging of Preclinical, Sporadic, and Autosomal Dominant Alzheimer's Disease. Neurotherapeutics. 2021. https://doi.org/10.1007/s13311-021-01026-5.

3. Peet B, Spina S, Mundada N, La Joie R. Neuroimaging in frontotemporal dementia: heterogeneity and relationships with underlying neuropathology. Neurotherapeutics. 2021.

4. Brooks DJ. Imaging Familial and Sporadic Neurodegenerative Disorders Associated with Parkinsonism. Neurotherapeutics. 2021. https://doi.org/10.1007/s13311-020-00994-4.

5. Schwarz AJ. The Use, Standardization, and Interpretation of Brain Imaging Data in Clinical Trials of Neurodegenerative Disorders. Neurotherapeutics. 2021. https://doi.org/10.1007/ s13311-021-01027-4.

6. Wilkenfeld DA, Orbell SL, Lingler JH. Ethical Considerations in Communicating Alzheimer's Disease Neuroimaging Biomarker Test Results to Symptomatic Individuals. Neurotherapeutics. 2021. https://doi.org/10.1007/s13311-021-01047-0.

7. Alosco ML, Culhane J, Mez J. Neuroimaging Biomarkers of Chronic Traumatic Encephalopathy: Targets for the Academic Memory Disorders Clinic. Neurotherapeutics. 2021. https://doi. org/10.1007/s13311-021-01028-3.

8. Goodman AM, Szaflarski JP. Recent Advances in Neuroimaging of Epilepsy. Neurotherapeutics. 2021. https://doi.org/10.1007/ s13311-021-01049-y.

9. Brandt SJ, Oral HY, Arellano-Bravo C, Plawecki MH, Hummer TA, Francis MM. Repetitive Transcranial Magnetic Stimulation as a Therapeutic and Probe in Schizophrenia: Examining the Role of Neuroimaging and Future Directions. Neurotherapeutics. 2021. https://doi.org/10.1007/s13311-021-01046-1.

10. McDonald BC. Structural Neuroimaging Findings Related to Adult Non-CNS Cancer and Treatment: Review, Integration, and Implications for Treatment of Cognitive Dysfunction. Neurotherapeutics. 2021.

Publisher's Note Springer Nature remains neutral with regard to jurisdictional claims in published maps and institutional affiliations. 